\title{
Spatially Distributed Naming Games
}

\author{
Luc Steels ${ }^{\dagger}$ \\ SONY Computer Science Laboratory Paris \\ 6 rue Amyot, 75003 Paris \\ France \\ VUB AI Laboratory \\ Pleinlaan 2, 1050 Brussels \\ Belgium \\ Angus McIntyre \\ SONY Computer Science Laboratory Paris \\ 6 rue Amyot, 75003 Paris \\ France
}

\begin{abstract}
The paper investigates the dynamical properties of spatially distributed naming games. Naming games are interactions between two agents, a speaker and a hearer, in which the speaker identifies an object using a name. Adaptive naming games imply that speaker and hearer update their lexicons to become better in future games. By engaging in adaptive naming games, a coherent shared vocabulary arises through self-organisation in a population of distributed agents. When the agents are spatially distributed, diversity can be shown to arise, and changes in population contact lead to language changes.

$K E Y W O R D S$ : self-organization, evolutionary linguistics, language contact, language change.
\end{abstract}

\footnotetext{
$\dagger$ Email: 'steels@arti.vub.ac.be'. More information about our research is available at $<$ http://ннr.csl.sony.fr/ $>$ and $\langle$ http://arti.vub.ac.be/>.
} 


\section{Introduction}

It is a fact that natural languages evolve at all levels: New sounds may get introduced or the sound system of a language may start shifting, new words and meanings may enter the lexicon existing meanings may expand or contract, new syntactic categories may emerge, syntactic structures may shift, features expressed through morphology may shift to a syntactic expression, etc. These changes may occur very rapidly, particularly in the case of contact languages such as pidgins and creoles (Arends et al., 1995). There may also be periods of relative stability, even in contact languages (Sebba, 1997). All these changes take place against a background of continuously changing populations (the linguistic community is in constant flux due to the in- and outflow of members) and changing environments and thus new sources of meaning (new artefacts, new institutions, new types of social relations, etc.).

If we restrict linguistic investigations to a single (idealised) speaker-hearer in a homogeneous language community who knows and speaks the language perfectly (Chomsky, 1975), it is unclear why languages should change. It is even more of a puzzle if we assume that language is to a large extent innate, which implies that true innovations can only occur through genetic mutations and subsequent spreading in the population (Pinker, 1994). With respect to the timescales observed in the emergence and spreading of linguistic innovations, genetic mutations are simply too slow. Moreover, the 'success' of a linguistic innovation is dependent on whether the group as a whole has adopted it. A mutation in a single agent does not give this agent any immediate advantage, and is thus unlikely to be favoured by natural selection.

On the other hand, if we accept that languages are invented and transmitted culturally, language change becomes self-evident. During transmission, and particularly during learning, variations will automatically arise. These variations may become conventionalised as they propagate to the rest of the group. Language learning, which is at the heart of the transmission process, then provides important clues about what kind of language changes are to be expected and conversely, changes in languages give us clues about the kind of learning processes that must be active. For example, language learners (as listeners) may overinterpret linguistic behavior, in the sense that they may erroneously believe that certain behavior is rule-governed. If learners then use this over-interpretation in subsequent language production, it can become a new norm. An empirical study of the kind of over-interpretation that has taken place may give us a clue towards the kind of learning strategies that were at work.

The cause of language changes may be internal to the language (Labov, 1994). For example, if a new sound starts to propagate, it may cause a chain shift of changes in existing sounds so that distinctiveness is retained in the total system. Other causes of language change are external (Nichols, 1992). They come from contact between languages due to increased communication between speaker communities, large-scale bilingualism causing mutual influence, the splitting of a community into separated groups allowing subsequent language divergence, etc.

This paper focuses on language change due to changes in the dynamics of the underlying populations, particularly in the communication structure between linguistic communities as determined by spatial location. A very basic linguistic 
behavior is investigated: the naming of objects with the aim of developing a common lexicon. The learning/construction procedure is kept as simple as possible so as to focus on the issue of language contact itself. The agents are spatially distributed so that the impact of changes in a community's structure on language can be studied. Even with many simplifying assumptions, we can see interesting phenomena emerge which cannot be explained by focusing on single speakers without learning capability.

The rest of the paper is in three parts. Section 2 introduces the linguistic behavior and the learning process of a single agent. In section 3 the typical behavior of such a system is studied without language contact. Finally, in section 4 an experiment related to the dynamics of language contact is discussed.

\section{The Naming Game}

The naming game (Steels, 1996) is a specific kind of language game which is played between two agents. One agent (the speaker) identifies an object by using a name. The game succeeds if the other agent (the hearer) agrees that this name is appropriate for the object identified. A naming game is adaptive when both participants in the game are able to change their rules (i.e. their wordobject associations) to be more successful in future games. The naming game is strongly related to other models for cultural lexicon acquisition as proposed by (Oliphant, 1996).

The naming game is a theoretical model for studying essential characteristics of language use and language formation, similar to the use of cellular automata as formal models of computation. In particular, naming games allow us to study the creation, propagation, and evolution of linguistic conventions. Naming is admittedly the simplest possible linguistic device imaginable but a lot can be learned that carries over to the creation, propagation, and evolution of more complex linguistic mechanisms such as phonologies or syntactic constraints. On the other hand, certain issues such as the emergence of ambiguity, multiple interacting levels, the grounding in real-world sensing or actuation, etc., cannot be studied with the very basic model presented here. Such issues can be studied with various extensions of the model as discussed in other papers. For example, Steels (Steels, 1997) describes games which relate perceptual features with words, Steels and Vogt (Steels and Vogt, 1997) discuss how language games can be grounded on physically embodied robots, and Steels and Kaplan (Steels and Kaplan, 1999) discuss the complex semantic dynamics in grounded robotic systems.

\subsection{Definition of the Naming Game}

We assume a set of agents $\mathcal{A}$ of size $\mathcal{N}_{\mathcal{A}}$ where each agent $a \in \mathcal{A}$ has contact with a set of objects of size $\mathcal{N}_{\mathcal{O}} \mathcal{O}_{a}=\left\{o_{0}, \ldots, o_{n}\right\}$. Some or all of the objects are shared between the different agents. A word is a sequence of letters drawn from a finite alphabet. The agents are all assumed to share the same alphabet. A lexicon $\mathcal{L}$ is a dynamical relation between objects, words and two quantities: The number of times the relation has been used and the number of times the relation was successful in use. Each agent $a \in A$ has his own lexicon $L_{a} \subset \mathcal{O}_{a} \times W_{a} \times \mathcal{N} \times \mathcal{N}$ which is initially empty. There is the possibility of synonymy and polysemy: A 
single word can be associated with several objects and a given object can be associated with several words. An agent $a \in \mathcal{A}$ can now be defined as a pair $a=\left\langle\mathcal{L}_{a}, \mathcal{O}_{a}\right\rangle$.

A naming game $N=\langle s, h, o\rangle$ is an interaction between two agents: a speaker $s$ and a hearer $h$ about a topic $o$ which is an object, $o \in \mathcal{O}_{s}$. The interaction proceeds as follows:

1 The speaker randomly selects a topic out of his set of objects. The speaker draws the attention of the hearer to the topic. In a natural conversation this could be by pointing or prior linguistic exchanges.

2 The speaker $s$ encodes the topic $o$ through a word $w$. The word chosen is the most successful word $w$ where $\langle o, w, u, s\rangle \in \mathcal{L}_{s}$. A word $w_{1}$ is more successful than a word $w_{2}$ iff $\left\langle o, w_{1}, u_{1}, s_{1}\right\rangle \in \mathcal{L}_{s},\left\langle o, w_{2}, u_{2}, s_{2}\right\rangle \in \mathcal{L}_{s}, u_{1} \geq u_{2} \geq 0$ and either $\left.s_{1} / u_{1}\right\rangle s_{2} / u_{2}$ or $s_{1} / u_{1}=s_{2} / u_{2}$ and $\left.u_{1}\right\rangle u_{2}$. If several words are equally successful, a random word is selected from the most successful set.

3 The hearer $h$ decodes the word $w$ to derive a set of objects $\mathcal{O}_{h}$ such that $o \in \mathcal{O}_{h} \Longrightarrow\langle o, w, u, s\rangle \in \mathcal{L}_{h}$

4 A naming game is successful iff $o \in \mathcal{O}_{h}$.

Several naming games involving different agents can be going on in parallel because interactions are always local.

An adaptive naming game is a naming game $N=\langle s, h, o\rangle$ as defined above, with changes to the lexicon of both the speaker and hearer as a side effect of the game. This means that we have to introduce a temporal dimension in the definition of an agent. An agent $a$ at time $t$ is defined as $a_{t}=\left\langle\mathcal{L}_{a, t}, \mathcal{O}_{a, t}\right\rangle$. A time point corresponds to an event in which two agents play a language game. If the speaker at time $\mathrm{t}$ uses a word $w$ where $\langle o, w, u, s\rangle \in \mathcal{L}_{s, t}$ and if the game ends in success, then $\langle o, w, u+1, s+1\rangle \in \mathcal{L}_{s, t+1}$ and $\langle o, w, u+1, s+1\rangle \in \mathcal{L}_{h, t+1}$. If a word is used by speaker or hearer but some failure has occured, then use is incremented but not success: $\langle o, w, u+1, s\rangle \in \mathcal{L}_{s, t}$ and $\langle o, w, u+1, s\rangle \in \mathcal{L}_{h, t+1}$.

The other changes defining the state of an agent at time $t+1$ are as follows:

1. Missing object (step 1 fails).

The object chosen as topic by the speaker is not shared by the hearer: $o \notin \mathcal{O}_{h, t}$. In that case, the game ends in failure but the hearer acquires knowledge about the object with an object propagation probability $p_{o}: \mathcal{O}_{h, t+1}=\mathcal{O}_{h, t} \bigcup\{o\}$.

2. The speaker does not have a word (step 2 fails).

In other words there is no $\langle o, w, u, s\rangle \in \mathcal{L}_{s, t}$. In that case, the game fails but the speaker may (randomly) create a new word $w^{\prime}$ and associate this with the object in his lexicon with a word creation probability $p_{c}: \mathcal{L}_{s, t+1}=\mathcal{L}_{s, t} \bigcup\left\{\left\langle o, w^{\prime}, 1,0\right\rangle\right\}$.

3. The hearer does not know the word (step 3 fails).

In other words there is no $\langle o, w, u, s\rangle \in \mathcal{L}_{h, t}$. In that case, the game ends in failure but the hearer may extend his lexicon with a word absorption probability $p_{a}: \mathcal{L}_{h, t+1}=\mathcal{L}_{h, t} \bigcup\{\langle o, w, 1,0\rangle\}$.

4. The topic is not decoded by the hearer (step 4 fails).

In other words, $o \notin H$. In that case the game ends in failure, but the hearer may acquire the word for his lexicon with a word absorption probability $p_{a}$ : $\mathcal{L}_{h, t+1}=\mathcal{L}_{h, t} \bigcup\{\langle o, w, 1,0\rangle\}$ 
If there is no explicit change as defined above, then the definition of an agent $a$ remains the same: $a_{t+1}=\left\langle\mathcal{L}_{a, t}, \mathcal{O}_{a, t}\right\rangle$

\subsection{Limitations AND assumptions of the NAMing Game}

The naming game as presented is almost trivially simple. It is not our intention to suggest it as a 'realistic' model of what happens in language formation. We do not claim that members of an emerging linguistic community spend their time playing naming games with each other in order to construct a language. Rather, we claim that activities similar to the naming game may form a part of the linguistic interactions that take place between different members of a community, or between parents and children. A mother who shows a child an object and says its name is playing a kind of naming game with the child, even though this is merely one among many possible kinds of interaction that may take place during the child's acquisition of language.

The naming game is a deliberate simplification, which is intended to illustrate or represent linguistic interactions rather than to accurately model them. Our main interest is in the mechanisms by which shared conventions may spread through a community, and the phenomena associated with this process. The simplicity of the naming game means that we can study these mechanisms and phenomena without the distractions and distortions introduced by a - probably vain - attempt to construct a highly 'realistic' model of linguistic interaction.

The naming game also involves a number of assumptions. It is important to make these explicit, so that we can either justify them - with reference either to the real world or to our particular focus of interest - or investigate the effects of relaxing or discarding our assumptions.

One major assumption made in this paper is that communication, both linguistic and non-linguistic, is reliable and unambiguous. Relaxation of this assumption is explored in (Steels and Kaplan, 1998), which shows that agent communities can in fact cope with high degrees of uncertainty, and that shared conventions may both emerge and persist under conditions of imperfect communication. Other assumptions relate to the capacity of the agents' memories - assumed to be reliable and effectively infinite. This assumption could also easily be relaxed, by imposing a limit on vocabulary sizes, introducing 'noise' into the agents' memories, and so forth. Informal exploration of this issue using a pruning mechanism that discards under-utilised words (thus effectively reducing lexicon size) suggests that the same phenomena described in this paper - convergence on shared vocabularies - will take place even when the agent's mental capacities are constrained in this way.

The naming game model also assumes that participants share the 'rules' of the game and a common interest in playing it. Their communication abilities are strong enough for them to coordinate their involvement in the game - including the signalling of success or failure - but not strong enough to allow them to resolve problems by dialogue. In defence of this assumption we would say that it is not our intention in this paper to explore the emergence of coordinated activity, as other work, particularly (Noble, 1998), has already done so. The question of how agents may come to engage in particular interactions is a very valid one, and one that would be well worth exploring experimentally. However, 


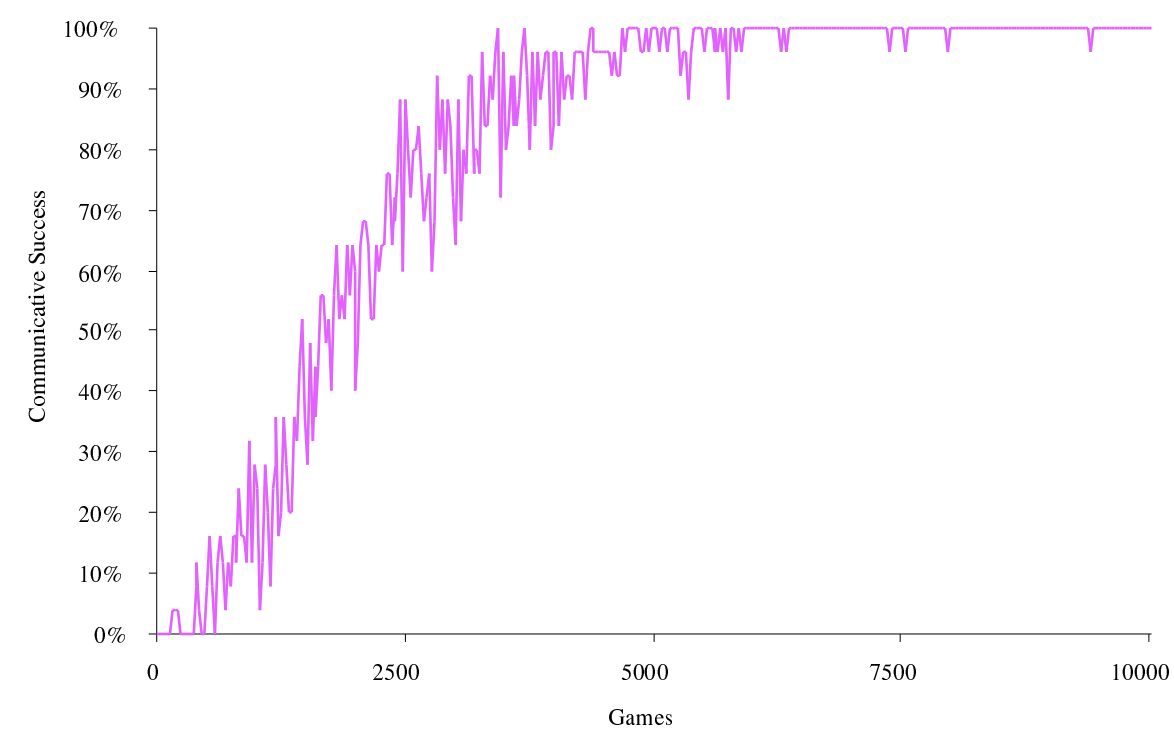

Figure 1: Simulation of closed system with 20 agents and 20 objects. Communicative success is shown on the $\mathbf{y}$-axis per 25 games. A rapid increase in communicative success is achieved for the first 5000 games, after which progress levels off to reach complete average success after 10000 games.

in this paper we are primarily interested in the formation of shared linguistic conventions once the basic interactional protocols have been established, rather than in the mechanisms that lead to the establishment of such protocols.

\section{Behavior}

\subsection{Behavior of Closed Naming Systems}

Let us now examine the behavior of naming games if played out in succession among a set of agents, where the choice of speaker and hearer is randomly chosen. First we look at a closed naming game system with the following parameters:

\begin{tabular}{ll}
\hline $\mathcal{N}_{\mathcal{A}}$ & 20 \\
$\mathcal{N}_{\mathcal{O}}$ & 20 \\
$p_{c}$ & 0.1 \\
$p_{a}$ & 0.75 \\
\hline
\end{tabular}

A typical simulation result after 10,000 games is seen in Figure 1. There is a rapid increase in a shared lexicon as seen from the communicative success.

An inspection of the lexicon of the invididual agents is quite interesting. Let us focus on agent a255. The size of the lexicon is 45 , which means that more than twice the number of words strictly required to cover the set of 20 objects 
have been either created or acquired by this agent. Here is the object-lexicon of agent a255. For each object we show the possible words followed by the use, success and score. The words are ordered according to the success criterion used by the agent to decide which word to prefer.

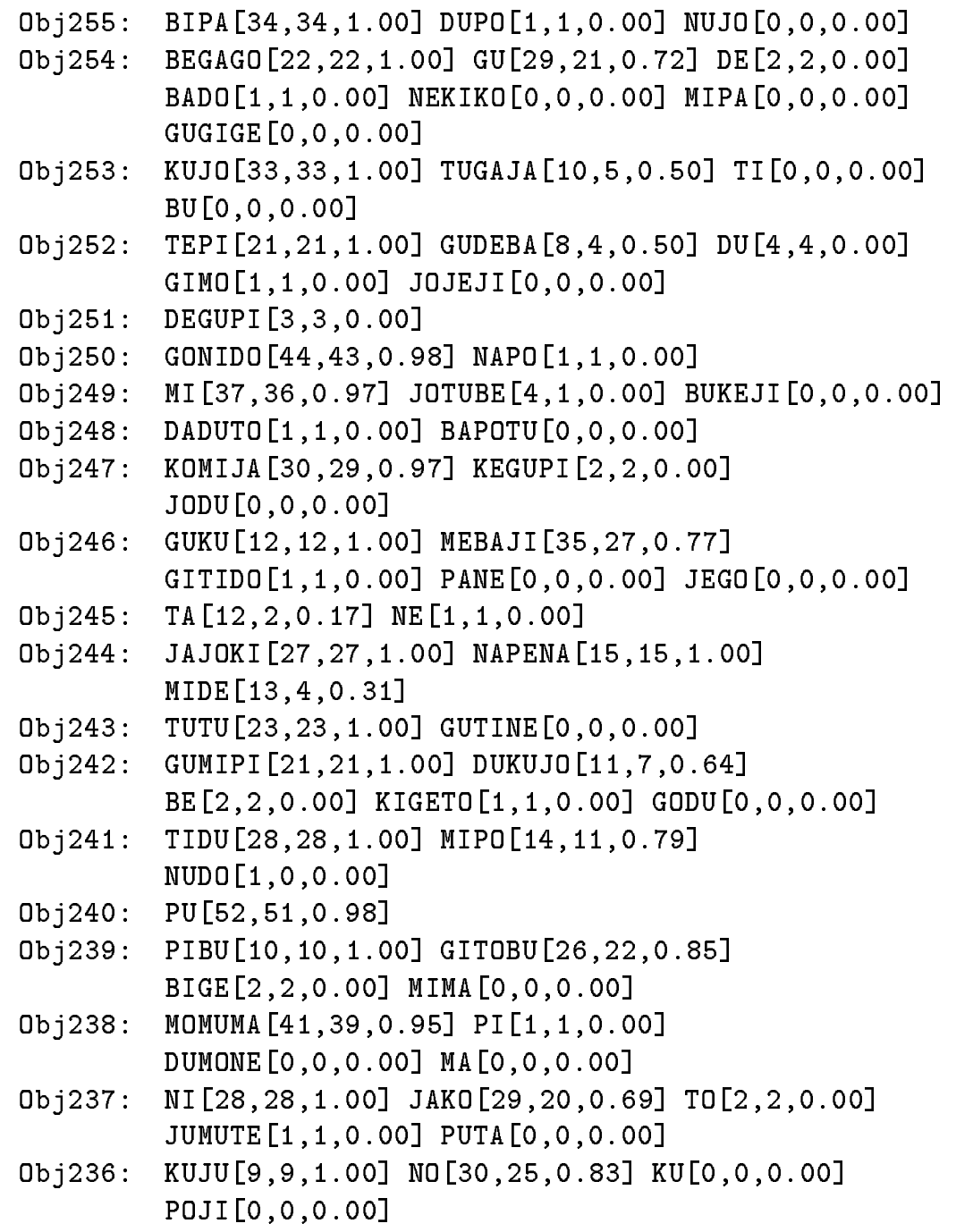

We observe that only a few objects have a single word (one of them is Obj240). For all others there is synonymy, with different words used to denote the same object. Strict synonymy is generally held to be rare in natural language: in our artificial language, the apparent synonyms are mostly redundant, with one form clearly dominating the others, and this dominance by a single word will be reinforced as the games proceed. Application of a simple pruning mechanism based on a criteria such as recency or frequency of use can then eliminate the unused synonyms. 
Let $S_{a}$ be the average synonymy in the lexicon of an agent $a$, which is in this case $S_{a 1}=3.40$.

In our simulations, homonymy and polysemy are very much rarer. In fact, polysemy, in which a single lexeme may have multiple meanings, is effectively absent in these simulations, as the only meanings in the world consist of atomic, unanalysed objects, allowing no possibility of the kind of analogical formations or meaning drift that give rise to polysemy. By contrast, polysemy is very much present in other simulations we have constructed, such as those described in (Steels and Kaplan, 1999).

Homonymy (following Lyons (Lyons, 1981) we take homonymy to be the case in which two or more unrelated lexemes have the same form but distinct meanings, e.g. the English words "bank" and "bat") is possible but rare, occurring only as a result of accidental generation of identical forms. This is seen in the following word-lexicon for the same agent. For each word the possible objects are printed, followed by the use and success counters. There are no homonyms in this vocabulary.

\begin{tabular}{|c|c|c|c|}
\hline JAJOKI : & Obj244 $[27,27]$, & GUG IGE : & $O b j 254[0,0]$ \\
\hline KEGUP I : & Obj247[2,2], & JEGO: & $\operatorname{Obj} 246[0,0]]$ \\
\hline JODU : & $\mathrm{Obj} 247[0,0]$, & MIPA: & $\mathrm{Obj} 254[0,0]$ \\
\hline GODU : & Obj242 $[0,0]$, & KUJU: & $\operatorname{Obj} 236[9,9]$ \\
\hline $\mathrm{DE}:$ & Obj254[2,2], & GIMO: & $\operatorname{Obj} 252[1,1]$ \\
\hline NI : & $0 b j 237[28,28]$, & NUDO: & Obj241[1,0] \\
\hline KUJO: & Obj253[33, 33], & PIBU: & $\operatorname{Obj} 239[10,10]$ \\
\hline MIDE: & Obj244 $[13,4]$, & GUT INE : & $\mathrm{Obj} 243[0,0]$ \\
\hline DUPO: & Obj255[1, 1], & NO: & $\operatorname{Obj} 236[30,25]$ \\
\hline DADUTO : & $\operatorname{Obj} 248[1,1]$, & MI: & $\operatorname{Obj} 249[37,36]$ \\
\hline GUDEBA : & Obj252[8,4], & BUKEJI : & Obj249[0,0] \\
\hline NUJO: & $\mathrm{Obj} 255[0,0]$, & TUTU : & Obj243 $[23,23]$ \\
\hline TUGA JA : & Obj253[10,5], & $\mathrm{BE}:$ & Obj242[2,2] \\
\hline MIPO: & Obj241[14, 11], & TO: & $\operatorname{Obj} 237[2,2]$ \\
\hline GUKU : & $\operatorname{Obj} 246[12,12]$, & TA: & $\operatorname{Obj} 245[12,2]$ \\
\hline BAPOTU : & Obj248[0,0], & MA: & $\operatorname{Obj} 238[0,0]$ \\
\hline GU: & Obj254[29,21], & PANE: & Obj246[0,0] \\
\hline KIGETO: & Obj242[1,1], & NEKIKO: & $\mathrm{Obj} 254[0,0]$ \\
\hline $\mathrm{NE}:$ & $\operatorname{Obj} 245[1,1]$, & MIMA : & Obj239[0,0] \\
\hline POJI: & Obj236[0,0], & BEGAGO : & Obj254[22, 22] \\
\hline JAKO: & $\operatorname{Obj} 237[29,20]$, & NAPENA : & Obj244[15, 15] \\
\hline PUTA : & Obj237[0,0], & NAPO: & Obj250 $[1,1]$ \\
\hline BU: & Obj253[0,0], & JUMUTE : & Obj237 $[1,1]$ \\
\hline GONIDO: & obj250[44, 43], & MOMUMA : & Obj238 $[41,39]$ \\
\hline BIGE : & Obj239[2,2], & KOMIJA: & $\operatorname{Obj} 247[30,29]$ \\
\hline DUMONE : & $\operatorname{Obj} 238[0,0]$, & PI: & Obj238 $[1,1]$ \\
\hline DEGUP I : & $\operatorname{Obj} 251[3,3]$, & $\mathrm{KU}:$ & $\operatorname{Obj} 236[0,0]$ \\
\hline JOJEJI : & Obj252[0,0], & DU : & $O b j 252[4,4]$ \\
\hline TEPI: & Obj252[21,21], & TIDU : & Obj241[28, 28] \\
\hline GITIDO: & Obj246[1,1], & BIPA: & Obj255[34, 34] \\
\hline BADO: & Obj254[1, 1], & JOTUBE : & $\mathrm{Obj} 249[4,1]$ \\
\hline
\end{tabular}




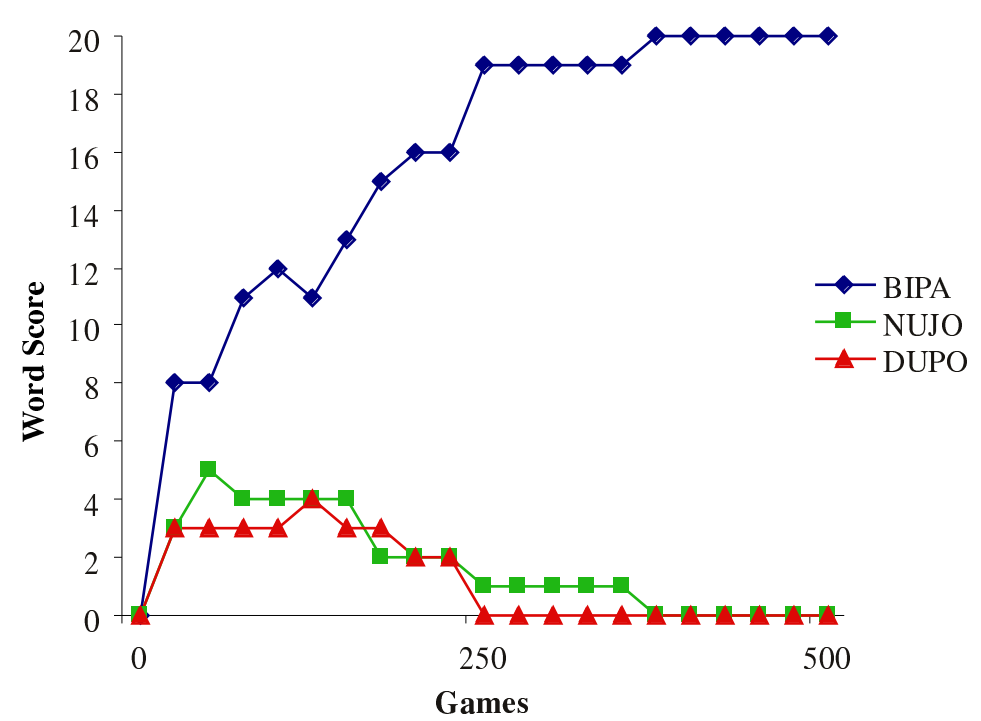

Figure 2: This figure shows the different competing words ("bipa", "nujo", "dupo") in the population for the same object. After a certain set of games, there is a convergence to a single word ("bipa").

$\begin{array}{llll}\text { DUKUJO: } & \text { Obj242[11,7], } & \text { GUMIPI : } & \text { Obj242[21,21] } \\ \text { MEBAJI: } & \text { Obj246[35,27], } & \text { GITOBU: } & \text { Obj239[26, 22] } \\ \text { TI: } & \text { Obj253[0,0], } & \text { PU: } & \text { Obj240[52,51] }\end{array}$

The rarity of homonymy in our simulations can be explained by the fact that homonymy can only occur when a particular word is independently created more than once. The probability that this will happen is low and depends on the word formation process: the size of the alphabet and the typical word length. We would expect homonymy

We define $H_{a}$ the homonymy in the lexicon of an agent $a$ to be equal to the percentage of the words that have more than one possible referent. $H_{a 1}=0$.

Let us now compare the lexicons of different agents. An agent understands many more words than it preferentially uses. Consequently average communicative success can reach $100 \%$ without the lexicons of the agents being the same. However we typically see a convergence towards a single word as illustrated in Figure 2 and thus the lexicons of the different agents will gradually become equal.

The convergence that is seen here is a special case of self-organisation in the sense of spontaneous formation of dissipative structures by the amplification and damping of random fluctuations (Prigogine and Stengers, 1984). It is a well known mechanism for achieving coherence without central control, and has been amply demonstrated in several biological systems for explaining pattern formation (Meinhardt, 1982) or in insect societies (Deneubourg, 1977). It applies here as follows: agents randomly couple words to meanings, and engage in communi- 


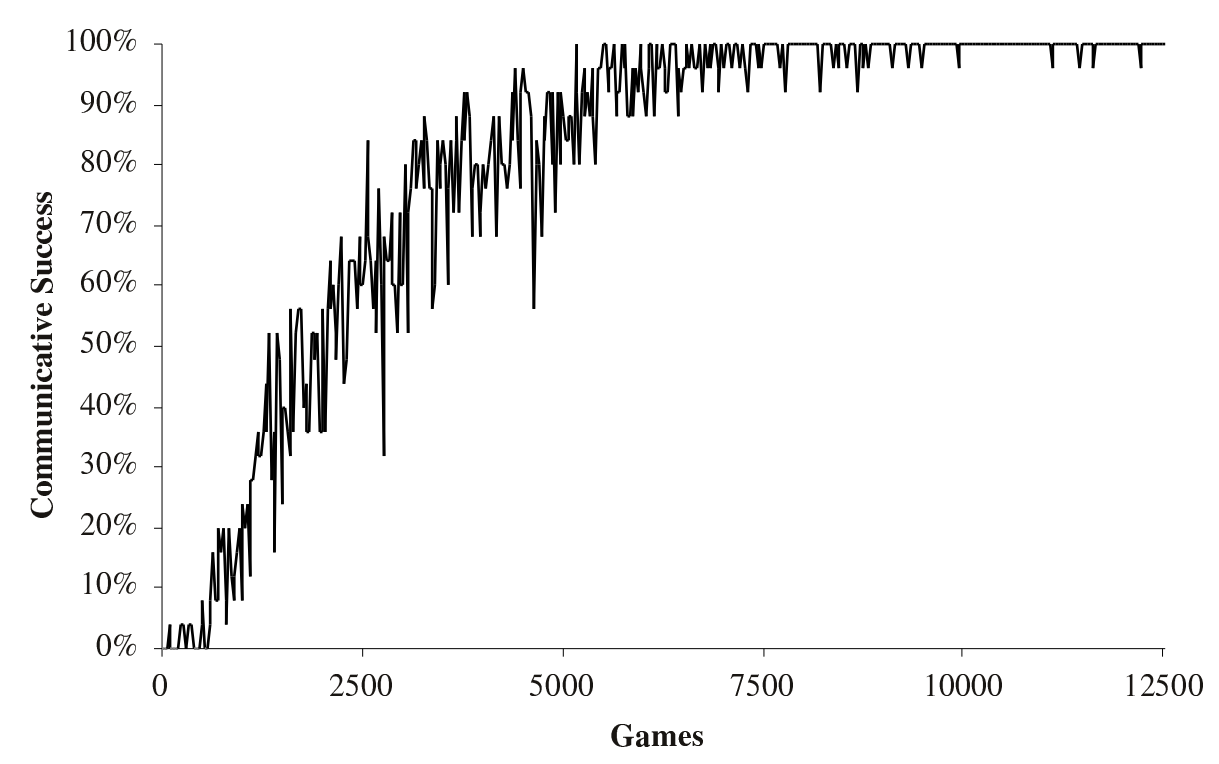

Figure 3: The population now consists of 50 agents and 50 objects. After scaling, we see the same curve shape as in 1 but now 12500 games were needed to reach total average communicative success.

cation. They record the success of a particular word-meaning pair and preferably use that in future communications. This establishes a positive feedback mechanism: a word that is used a lot will have a high communicative success and will hence be used even more.

Changes in the main parameters of the system lead to the following observations:

1 Increase in the size of the population $\mathcal{N}_{\mathcal{A}}$ will obviously increase the number of games that is needed before communicative success (and coherence) is reached. This is seen in Figure 3 which shows a series of language games with a population of 50 agents.

2 Increase in the number of objects $\mathcal{N}_{\mathcal{O}}$ will likewise increase the number of games, as shown in 3 . Repeating the simulations with progressively larger agent populations or object sets leads to longer times to reach communicative success, but the phenomena described in this paper appear to hold irrespective of population size. Kaplan, Steels and McIntyre (Kaplan et al., 1998) demonstrate that complete communicative success can be achieved even for large - 1000 agents or objects - populations or meaning sets. The time taken to reach $100 \%$ success increases as a function of population size, with the success curve assuming a more pronounced sigmoid shape as the population is increased. This type of curve, with a flat initial section followed by a steep climb followed in turn by a gradual levelling off, is commonly seen in models of growth, and has been widely observed in the 


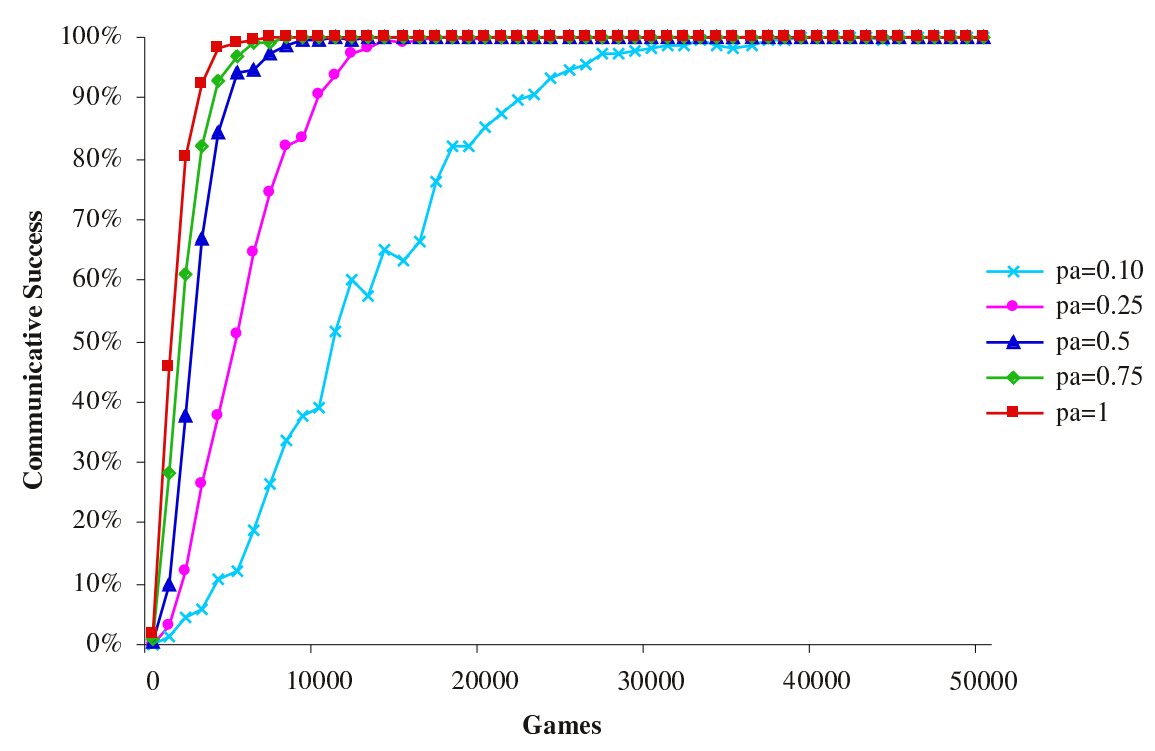

Figure 4: The figure plots the result of language games with 20 agents and 20 objects, each time varying the word absorption rate. As this rate increases, the time needed to reach communicative success increases. Note that the size of the gameset (1000 in this figure but 25 in figure 3) determines the amount of detail of the graph. Smaller variation is smoothed in these graphs because the size of the game set is larger.

propagation of linguistic conventions in human populations (Kroch, 1989; Niyogi and Berwick, 1995).

3 An increase in the probability of word creation $p_{c}$ has more subtle effects. We redo the first experiment (with 20 agents and 20 objects) but change $p_{c}=0.75$. It takes noticeably longer to reach communicative success. More importantly, the agents create many more words $S_{a 1}=6.15$ compared to 3.40 previously. A typical example is the following set in one agent for one object:

$$
\begin{aligned}
& \text { Obj701: } \operatorname{MAPO}[32,32,1.00] \operatorname{KEMI}[25,15,0.60] \operatorname{JEDA}[3,3,0.00] \\
& \text { DA }[2,2,0.00] \text { JAGEPO }[2,2,0.00] \operatorname{JOKE}[1,1,0.00] \\
& \text { BUTAJU }[0,0,0.00] \text { BI }[0,0,0.00]
\end{aligned}
$$

4 An increase in the probability of word absorption $p_{a}$ has an effect as seen in Figure 4 . As the word absorption rate decreases - in a real world situation, this might be due to the time needed to learn the form of the word or increased background noise - it takes longer to reach steady communicative success. 


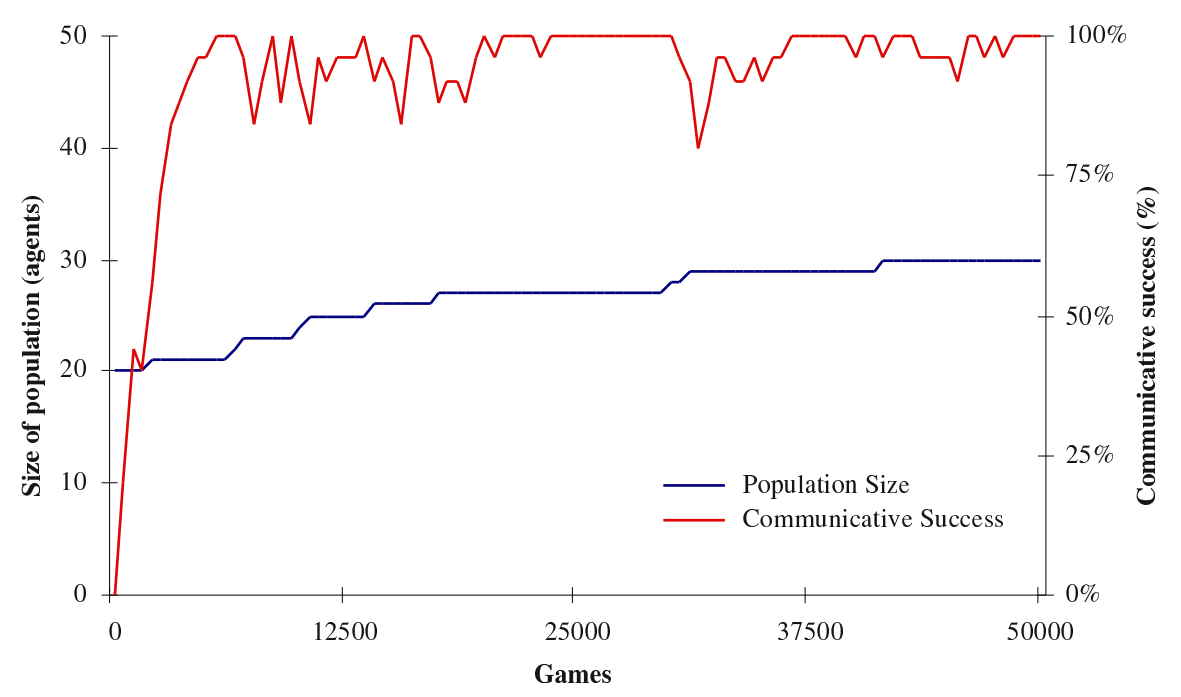

Figure 5: The figure plots the result of open naming games starting with a population of 20 agents and increasing the population with probability $p_{e} \mathcal{A}=0.00025$. The (average) communicative success per 500 games is shown on the y-axis. The population is able to cope with the continuous extension.

\subsection{Behavior of Open Naming Systems}

We now turn to an investigation of the naming game as an open system. This means that there is an in- and outflux of agents and objects. We can study both aspects at the same time by making the set of objects equal to the set of agents, i.e. the agents only talk about each other. The set of agents and objects now have a temporal dimension: $\mathcal{A}_{t}$ denotes the set of agents at time $t$ of size $\mathcal{N}_{\mathcal{A}}, t$ and $\mathcal{O}_{a, t}$ denotes the set of objects to which $a$ has access at time $t$. For the purposes of the experiments below, $\mathcal{A}_{t}=\mathcal{O}_{a, t}$ for every $a \in \mathcal{A}_{t}$.

Let $p_{e} \mathcal{A}$, the entrance probability, denote the probability that a new agent may enter population $\mathcal{A}$ and $p_{d} \mathcal{A}$, the departure probability, the probability that a new agent may leave the population $\mathcal{A}$. The entrance of a new agent has two effects: (1) The new agent has no lexicon yet. It has to acquire the lexicon that exists already in the population and may introduce new words himself. (2) The new agent also constitutes a new object, so the others have to invent and agree upon a new word to name the new agent. The departure of an agent has also two effects: (1) The agent can no longer be the topic of a conversation and so a word falls into disuse. (2) The agent takes with him a particular lexicon and so the collective memory diminishes.

Here are the result of some simulations showing the effect of different entrance and departure probabilities. 5 shows the effect with a low $p_{e} \mathcal{A}=0.00025$. We see that each time when the population extends the communicative success drops but the agents recuperate by developing the necessary vocabulary to successful name the new agents. 


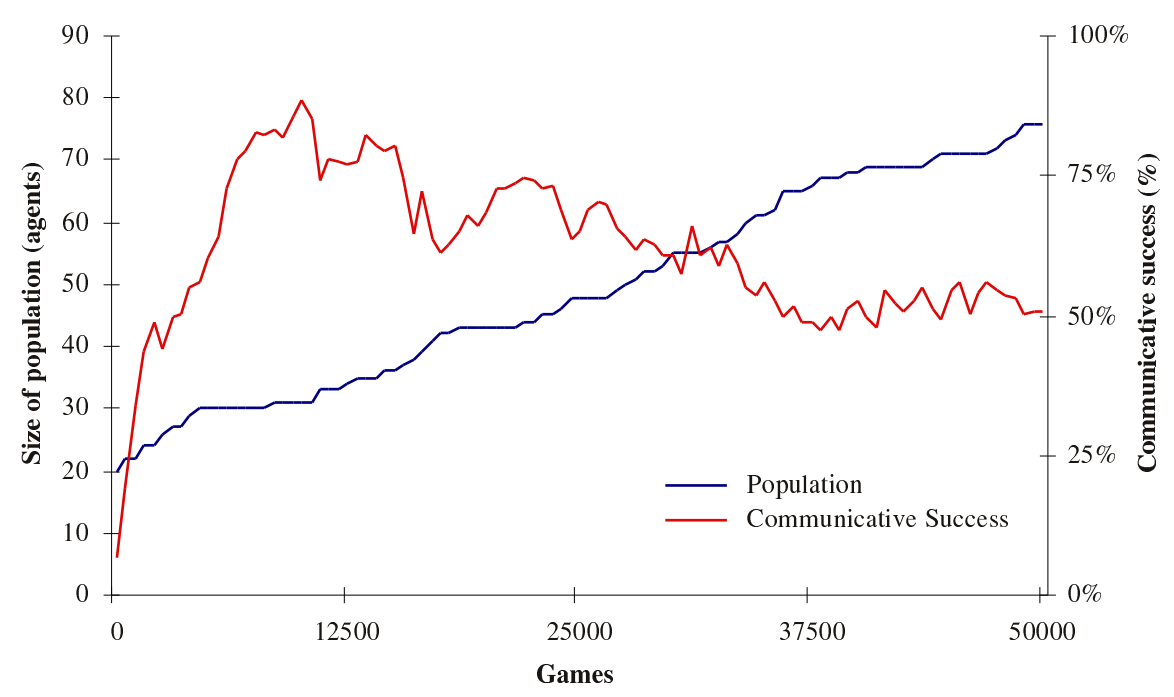

Figure 6: The figure starts with a population of 20 agents which increases with probability $p_{e} \mathcal{A}=0.001$ to reach around 80 members at the endpoint. The (average) communicative success per 500 games is shown on the y-axis. The population is not able to cope with the continuous expansion.

The next experiment, shown in Figure 6, shows the effect with a high entrance probability $p_{e} \mathcal{A}=0.001$. Now the population is not able to keep up with the influx of new agents (and hence new objects)

Next we look at the departure probability. Figure 7 shows the effect with a departure probability $p_{d} \mathcal{A}=0.001$. Agents leave the population but this does not cause a loss in communicative success because the remaining agents have an easier task (there are less objects) and the lexicon is sufficiently preserved in the rest of the population.

In natural populations there is usually a positive entrance and departure rate. Figure 8 and Figure 9 study the impact of this flux. Figure 8 shows what happens with low probabilities. Although the members of the population change and therefore also the objects they talk about change, there is no loss in communicative success which means that the population is able to cope both with in- and outflux of agents and in- and outflux of objects.

Figure 9 shows what happens with higher entrance and departure probabilities. Every time the population of agents (and thus of objects) goes up there are losses in average communicative success. When the population decreases the group catches up but this may not be sufficient to reach total communicative success. 


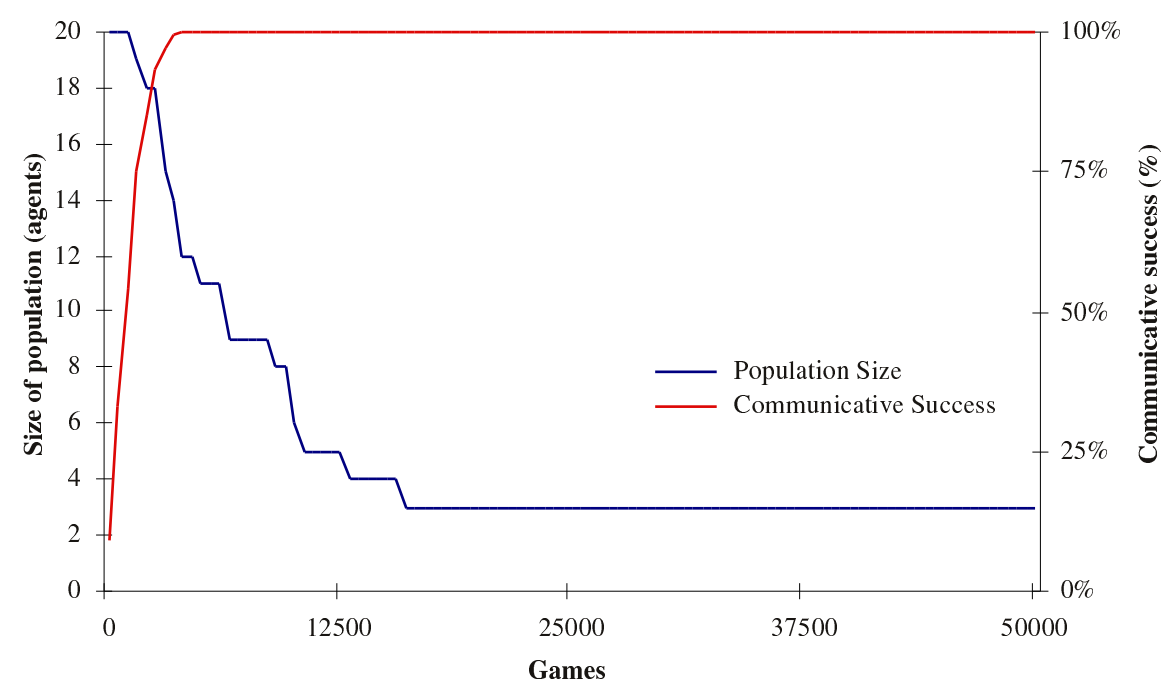

Figure 7: The figure starts with a population of 20 agents which decreases with departure probability $p_{d} \mathcal{A}=0.001$. The (average) communicative success per 500 games is shown on the $\mathbf{y}$-axis. Although the population dies out, there is no loss in communicative success.

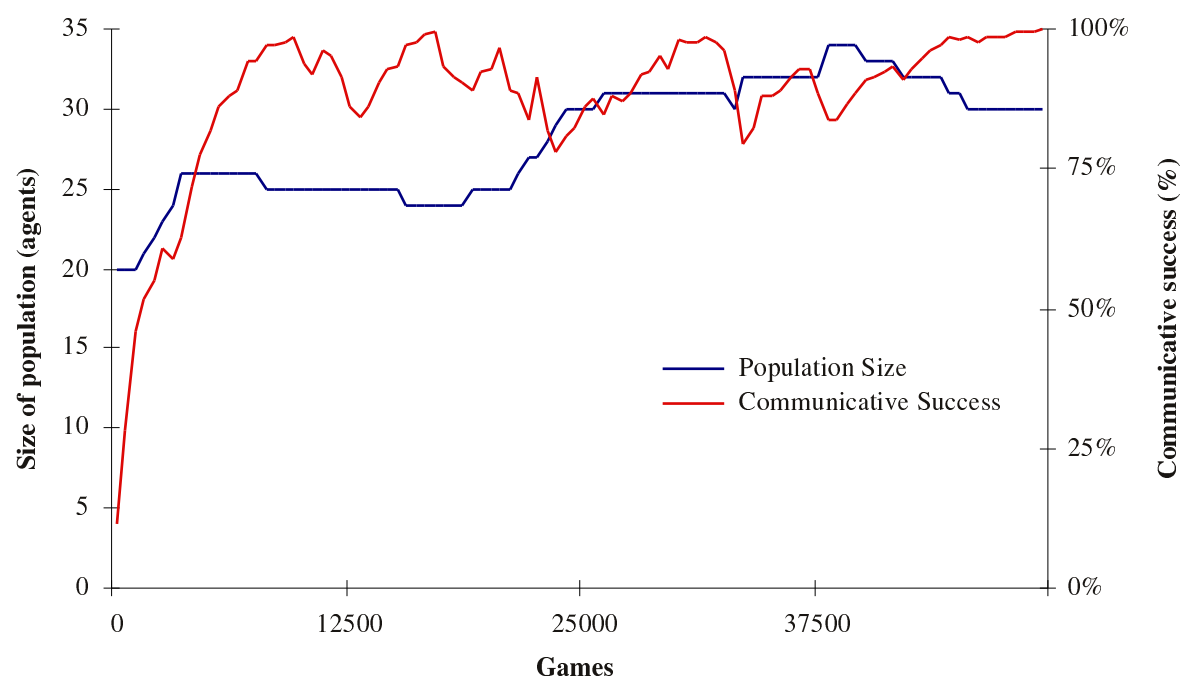

Figure 8: The figure has positive probabilities for entrance $p_{e} \mathcal{A}=0.0005$ and departure $p_{d} \mathcal{A}=0.0005$. The population is able to cope with the flux. 


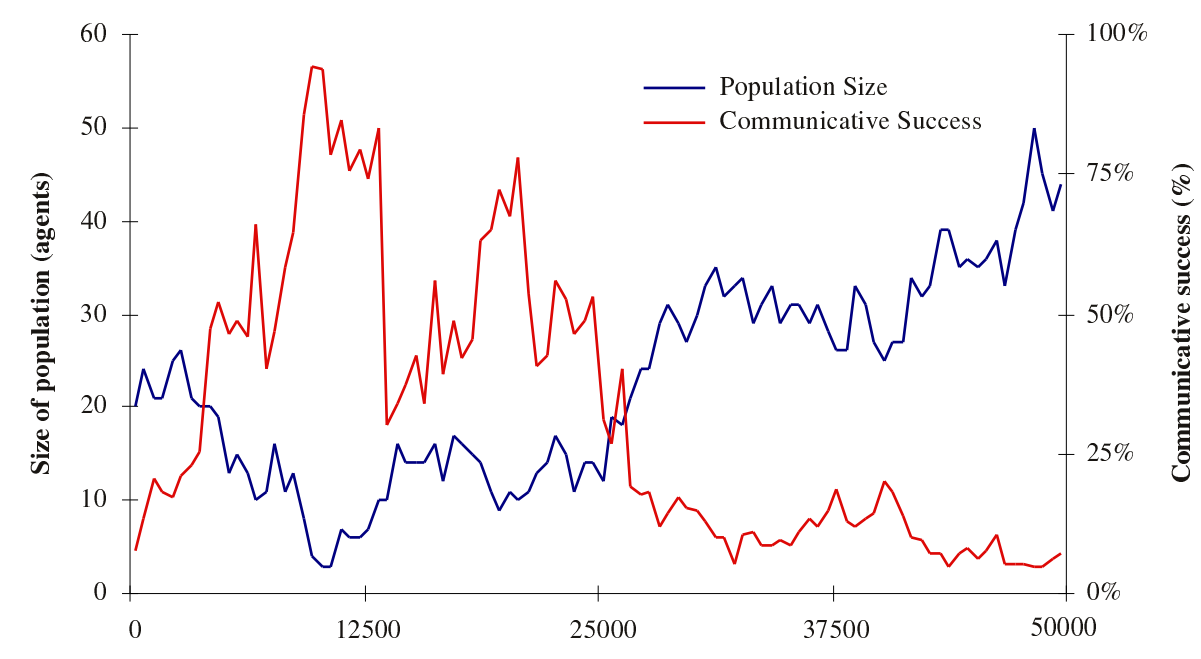

Figure 9: The figure has higher positive probabilities for entrance $p_{e} \mathcal{A}=0.01$ and departure $p_{d} \mathcal{A}=0.01$. The population is no longer or barely able to cope with the flux. A rich dynamics is seen.

\section{Language contact}

\subsection{Spatial Structures}

It is clear that in the evolution of natural languages the spatial distribution of the agents plays a very large role (Nichols, 1992). This can also be modeled computationally and mathematically by locating the agents in a 2-dimensional space. In our experiment, the space is taken to represent a physical space, thus modeling geographical distribution of agents. It might equally well represent a social, genetic or economical space. We could even perform experiments in a multi-dimensional space representing several of these alternate dimensions.

Each agent $a$ has $\mathrm{x}, \mathrm{y}$ coordinates $a_{x}, a_{y} . d_{a, b}$ is the trigonometric distance between two agents $a$ and $b$ :

$$
d_{a, b}=\sqrt{\left(a_{x}-b_{x}\right)^{2}+\left(a_{y}-b_{y}\right)^{2}}
$$

A spatial distribution is randomly generated, modulated by a clustering tendency. A typical example of a distribution is given in Figure 10.

The probability with which two agents $a$ and $b$ interact is based on their respective distance, and an interaction factor $m_{a}$, which determines the weight of the distance. If the interaction factor increases, the role of distance becomes higher and interactions tend to reflect the spatial clustering more. Based on this parameter we can study the evolution of language when communications between the communities increase. Our simulations show that average communicative success evolves as before (see Figure 11). However this obscures the interesting phenomena which can only be seen when inspecting and comparing the languages 


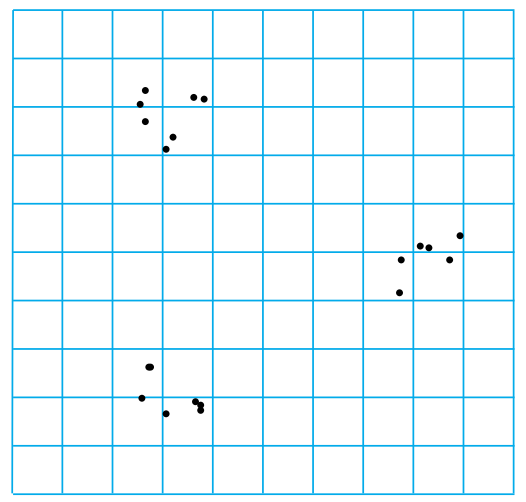

Figure 10: The figure shows the spatial distribution of a set of 20 agents. There is clustering around three centers.

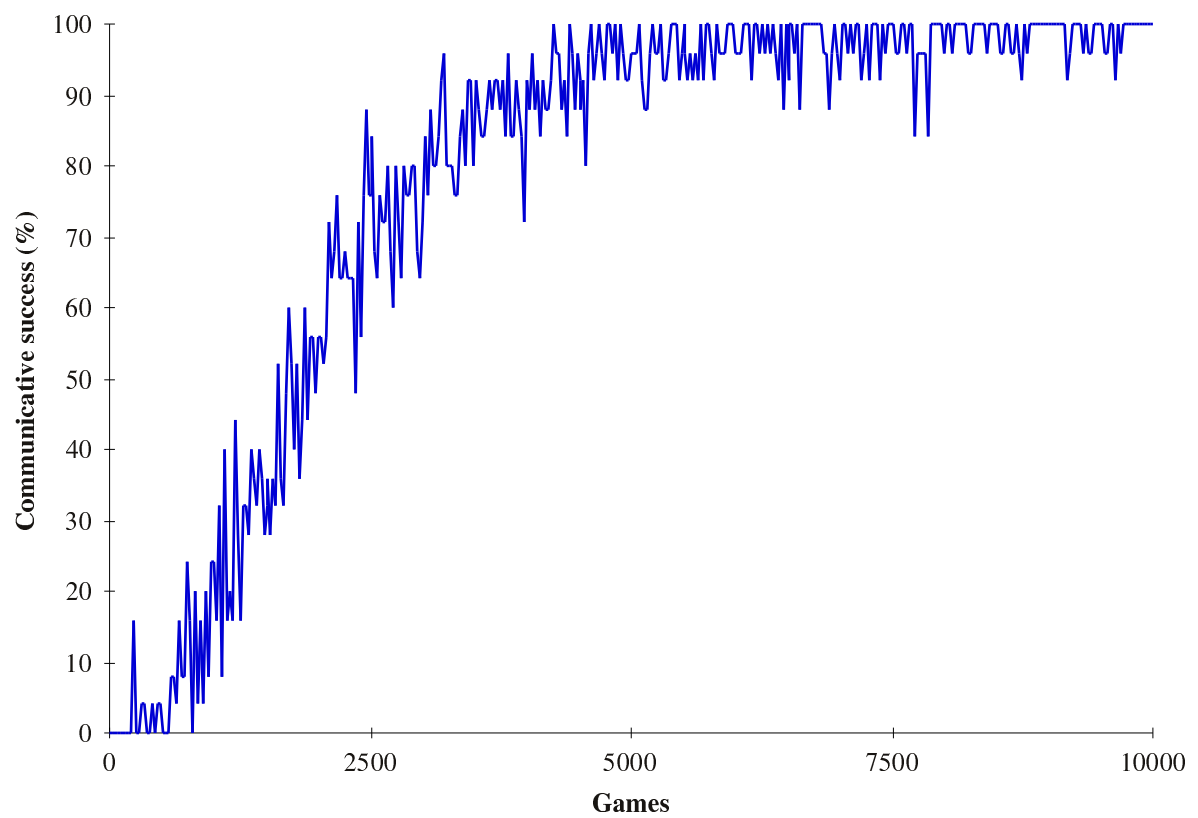

Figure 11: The figure shows the evolution of communicative success in a group of 20 agents clustered in three communities. Success is never total because there are occasional interactions with members of the other community, however intercluster communication reaches total success. 
of the different agents. Inspection of the agent lexicons reveals that agents will develop a stable language within their cluster. But they will also develop a second language, an interlingua which is weaker but shared among the different clusters. This interlingua will become stronger as more agents interact between clusters. Thus we observe language diversity due to the spatial distribution but at the same time occasional cognates as well as a second interlingua.

The following vocabularies illustrate this point clearly. The first vocabulary is taken from an agent from the leftmost cluster in Figure 10:

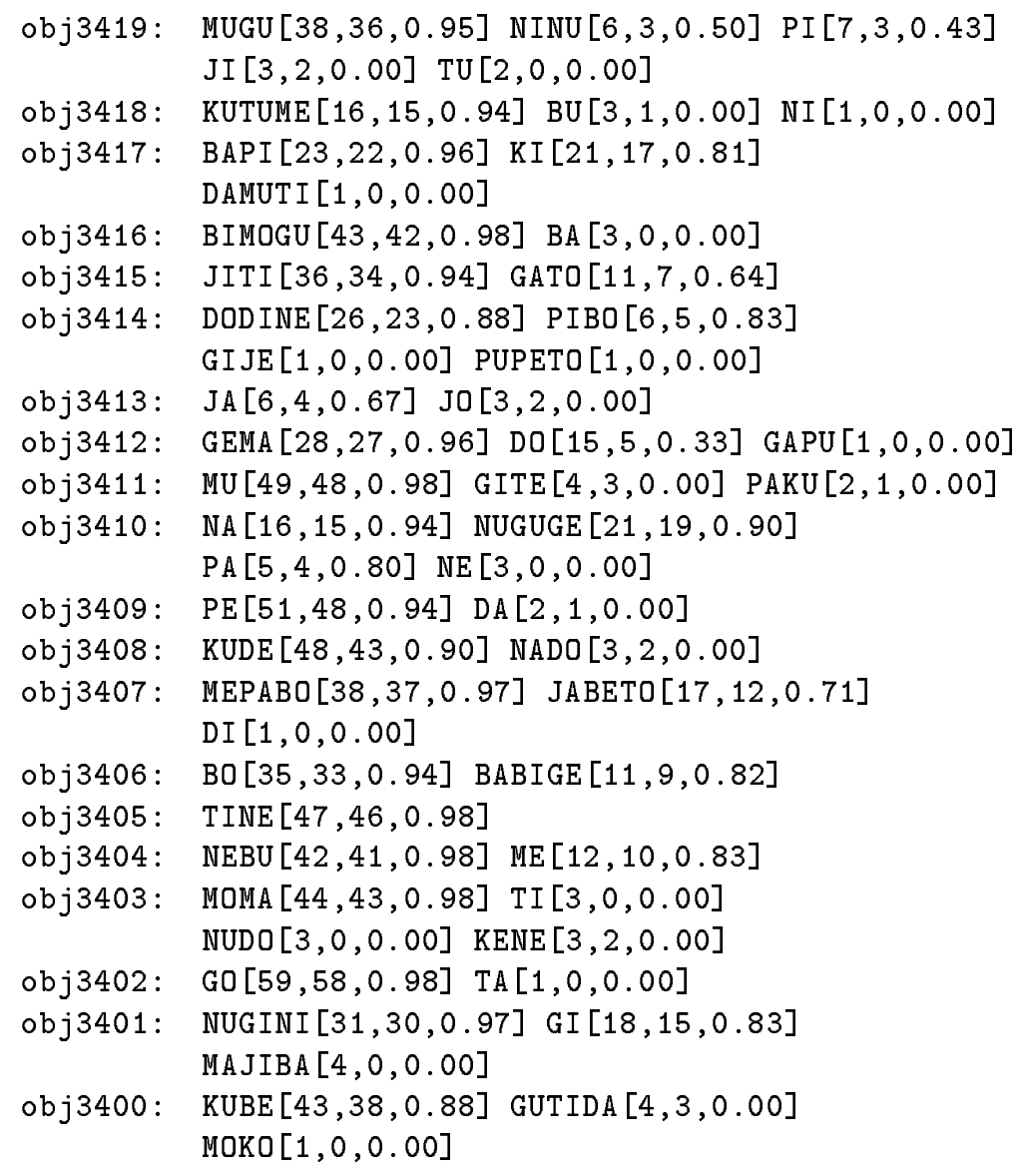

This is the vocabulary for an agent taken from the rightmost cluster in Figure 10:

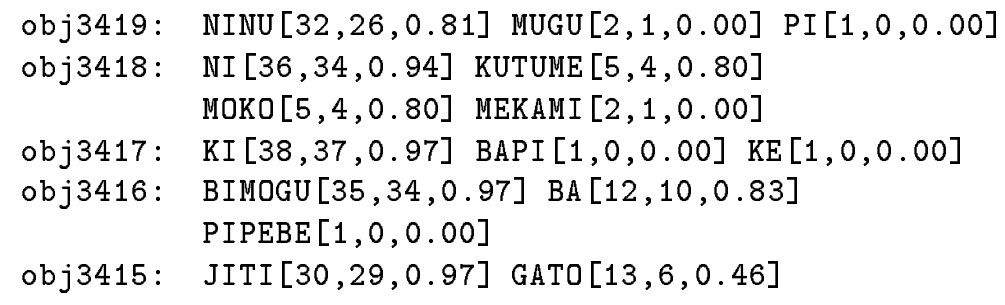




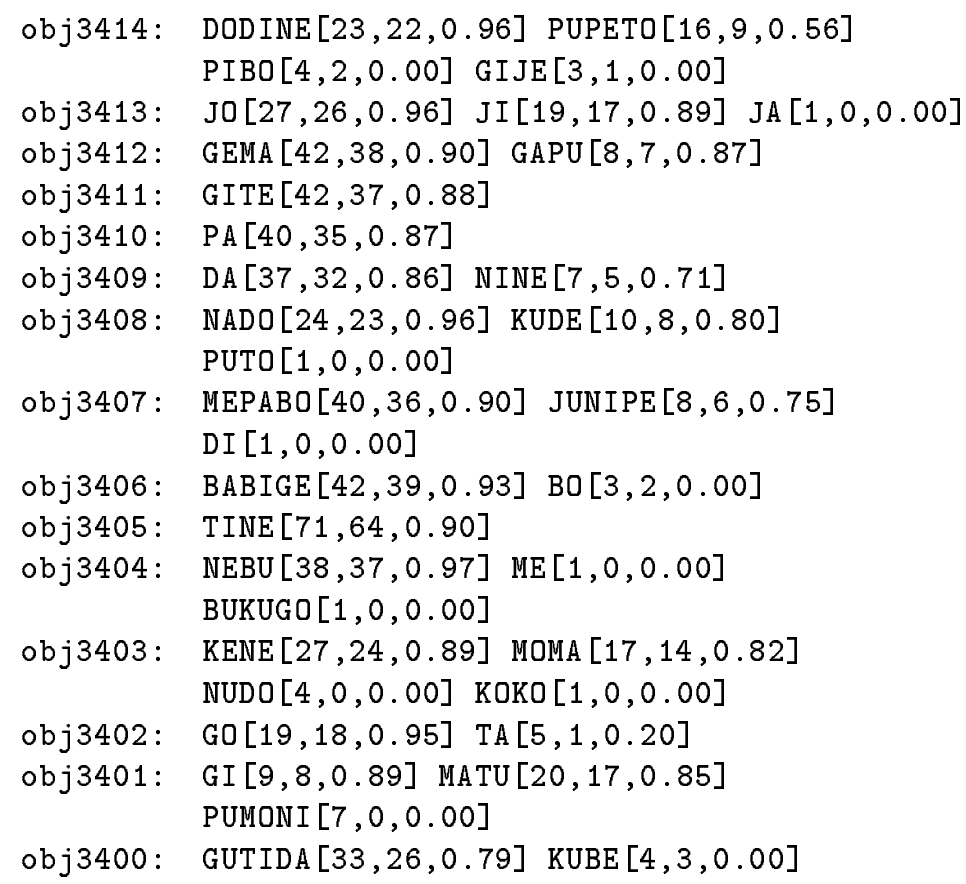

Some words (for example "TINE" for obj3405) are shared. But most of the time there are at least two words. One word is used preferentially inside the cluster, the other is known but preferentially used by members of another cluster. Thus the word "MUGU" for obj3419 is preferred for the first object in the first cluster and known but not preferentially used by the agent in the second cluster. Conversely, "NINU" is preferred for the same object by the agent in the second cluster, although he also knows "MUGU".

When the interaction factor increases, we see further differentiation because there is less communication between clusters. When it is decreased, we see more coherence because there is more intercluster communication. Thus we can effectively tune divergence or coherence based on probability of interaction between communities (clusters) of agents.

\subsection{The Dynamics of Language Contact}

This brings us to the core subject of the present paper. Given a spatial distribution and a variable degree of interaction, we can study the impact of increased communication and hence more intense language contact on clusters of language communities.

The experiment runs as follows. Agents in three different clusters (a, b, and c) interact and evolve a lexicon for each cluster. The interaction between clusters is initially very weak (see Figure 12). At some point in time the intercluster communication is increased drastically. At first there is a drop in communicative success but then total communicative success is again reached.

This general evolution hides however the more interesting developments. Figure 13 shows the evolution of coherence $C_{\mathcal{A}, t}$ for each cluster (a,b, c) separately 


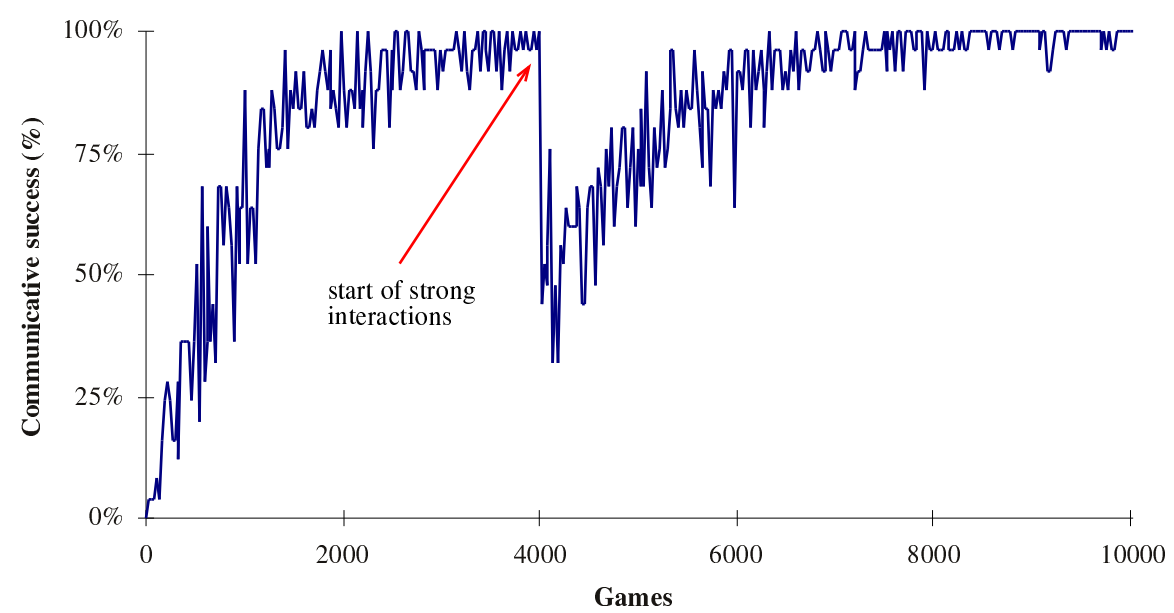

Figure 12: Evolution of average communicative success in a Group of agents with first weak and then strong interactions. The average communicative success per 25 games is shown at each point.

and also for the total set of agents. As long as the agents have relatively little contact, total coherence is low although coherence of each cluster is high. Coherence starts to increase with increased contact. Coherence in each cluster diminishes somewhat because the agents in the cluster are in the process of accomodating to the total set.

Figure 14 shows with the same scale the evolution of the equality measure $E_{\mathcal{A}_{1}, \mathcal{A}_{2}, t}$ between clusters a and b, a and c, and b and c. We see that the equality reaches a plateau before the increase in contact but then starts to climb. This means that the languages of the different groups are in the process of merging due to the increased language contact.

Finally, mutual understanding or bilingualism is shown in the next figure (Figure 15). The moment language contact increases we see a rapid increase in understanding, i.e. in how far a prefered word of one agent is also known to the other agent.

Thus we see that increased contact causes at first a rapid increase in bilingualism, then a gradual mixing of the languages, and, if the contact continues, an evolution towards complete coherence. The more rapid the contact is increased, the faster the three phases can be observed. Interestingly enough the communicative success of the whole system (intra-cluster and inter-cluster communication) is hardly affected, as shown in Figure 12.

These various phases are also seen in natural languages (Thomason and Kaufman, 1988). A common phenomenon is 'borrowing' of lexical items between adjacent language communities. Examples of this include central Belgium, where two language communities (French and Flemish) co-exist and large groups of the population have become bilingual. In these cases we see a strong mutual 


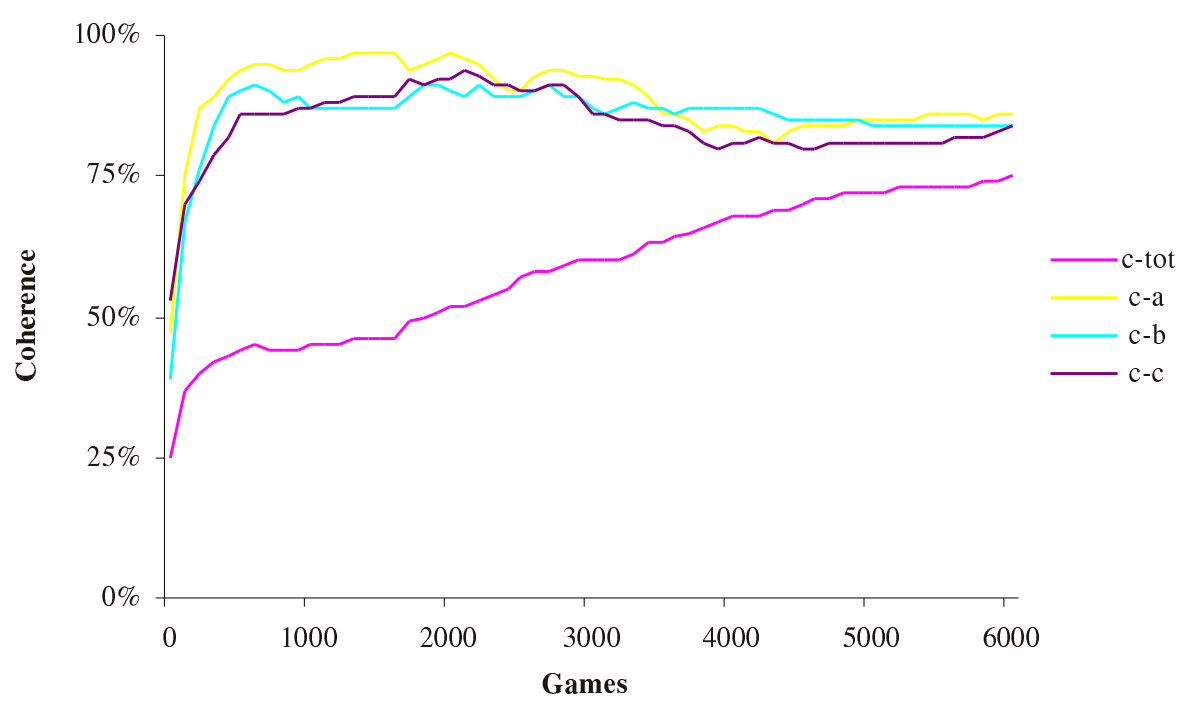

Figure 13: The figure shows the evolution of coherence plotted for every 25 games. We see that initially each individual cluster reaches a high level of coherence, whereas the total set of agents has a much lower coherence. When contact between the groups is increased, global coherence begins to rise steadily.

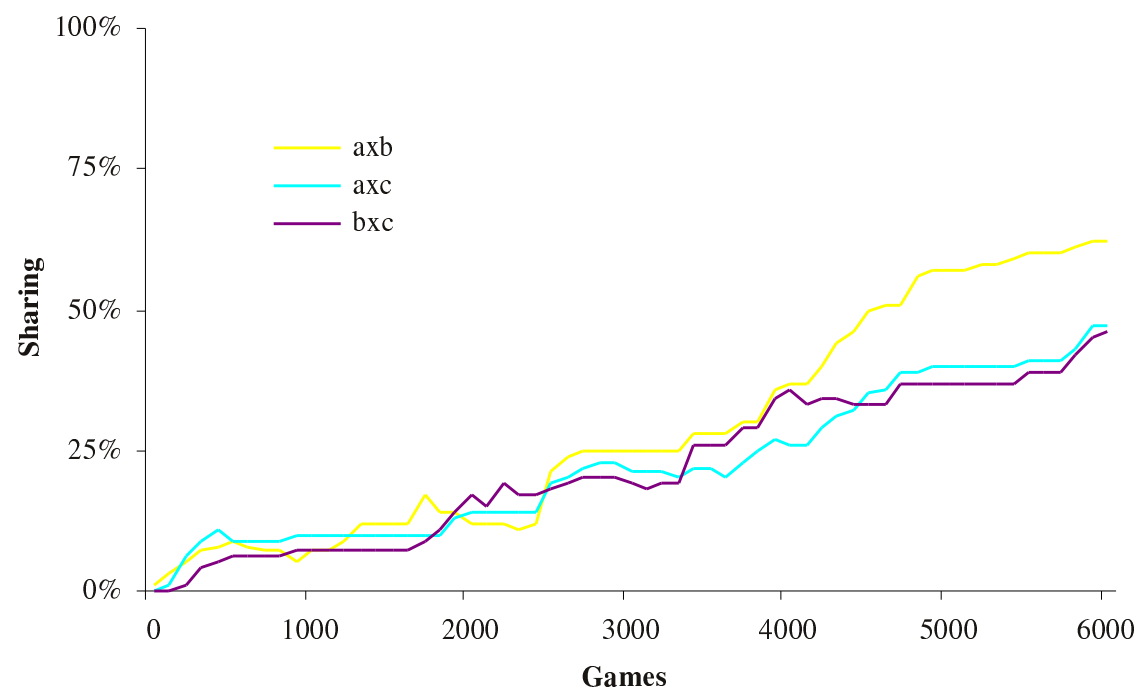

Figure 14: The figure shows the evolution of how many prefered words are shared between groups of agents. This starts to increase slowly after language contact is increased (at the 1500 game mark). 


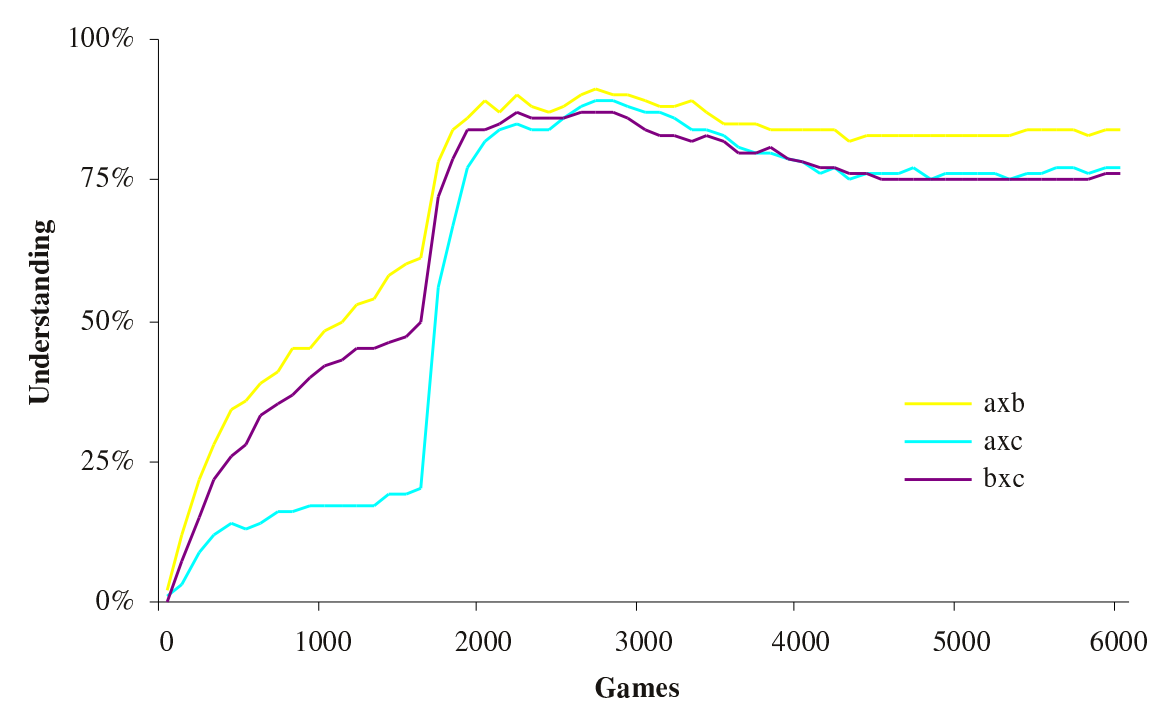

Figure 15: Evolution of intercluster understanding or bilingualism. A rapid increase is seen after agent contact is increased (at the 1500 game mark).

influence on the lexicons of both communities. Thus a cup will be called "kop" in Dutch but "tas" in Flemish, influenced by the French "tasse". This occurs despite the existence of a homonymous form "tas", meaning bag in Dutch as well as Flemish.

A less-balanced situation may occur if one group is numerically or politically stronger than another. A subpopulation that has been exposed due to invasion or migration to a larger and more dominant language community may progressively or radically shift their lexicon towards the language of the majority group, even though parts of the speakers' own language, such as the grammar, may remain intact. Such phenomena are observed in the formation of pidgin and creole languages: Sebba (Sebba, 1997) describes the concept of a lexifier, typically the language of the dominant group, which constitutes the target towards which the evolving language develops.

\subsection{FURTHER WORK}

Clearly, there is a great potential for further experimentation. Experiments which could usefully be conducted include:

1 Population sizes could be varied to see in how far population domination leads also to language domination.

2 Other factors could be made to influence the probability of inter-cluster communication. For example, the social distance could have the same stratification effect as spatial distance. 
3 Rather than increasing language contact, we could decrease it, showing that the population diversifies.

4 The populations in the cluster experiment above are closed. Another obvious experiment is to let them vary at specific rates, so that the languages in each cluster are moving targets.

All these experiments reflect situations that occur in human language populations and can thus be compared to language evolution phenomena that have been observed to accompany population changes.

\section{Conclusion}

We believe that language is a complex adaptive system which self-organises and achieves coherence even if the agents or possible meanings change. Language evolves partly due to its own internal dynamics and is transmitted culturally and not genetically. These hypotheses differ substantially from the notion that language is primarily transmitted genetically through an innate language acquisition device embodying universal grammar, and hence originated due to genetic evolution (Pinker, 1994). Our work resonates strongly with research on historical linguistics (Nichols, 1992), sociolinguistics (Labov, 1994), and the study of language contact and language formation as observed in pidgin and creole languages (Arends et al., 1995).

We propose that spatially distributed language games provide theoretical frameworks and associated simulation tools to study the issues raised in these fields. For example, our experiments demonstrate that language convergence does not necessarily have to be caused by a common origin. The fact that languages share vocabularies, as investigated in statistically oriented research towards cognates (shared words) (Ruhlen, 1994), can be the result of interaction between clusters of language users, and is not necessarily the result of a universal common Ursprache as comparative linguists commonly assume. It has also been demonstrated that diversity is not necessarily due to a progressive mutation of an existing language when a subgroup has become isolated, but can be explained in terms of the independent evolution of lexicons in isolated clusters. Relative coherence in such cases follows only after subsequent contact between clusters. It should be possible to gain useful results in this area by feeding the simulations with data from human population change and empirical data on lexical evolution.

The main goal of the paper was to show through a series of simulation experiments that naming game systems, particularly open and spatially distributed games, exhibit a rich and interesting dynamics. This paper has only scratched the surface and many more variations can and will be examined, including age structure, bilingualism, spatial migrations, etc. Moreover it is clearly possible to develop a mathematical theory of naming game systems, so that the relations between the different parameters become understood, similar to comparable studies in cellular automata or evolving populations. This remains work for the future and we hope other researchers will be encouraged to join us in this exciting venture. 


\section{Acknowledgement}

This work was financed and carried out at the Sony Computer Science Laboratory in Paris. We are indebted to Toshi Doi and Mario Tokoro to be able to work in this superbly productive environment. The BABEL software tool under development by Angus McIntyre (McIntyre, 1998) at SONY CSL-Paris has proved invaluable for efficiently exploring the issues discussed in this paper.

\section{References}

Arends, J., Muysken, P. and Smith, N. Pidgins and creoles: an introduction. Amsterdam: Benjamins (1995).

Chomsky, N. Reflections on Language. New York: Pantheon (1975).

Deneubourg, J.-L. Application de l'ordre par fluctuations à la description de certaines étapes de la construction du nid chez les termites. Insectes Sociaux 24 (2), 117-130 (1977).

Fasold, R. and Schiffrin, D. Language Change and Variation. Washington, DC: Georgetown University Press (1989).

Kaplan, F., Steels, L. and McIntyre, A. An architecture for evolving robust shared communication systems in noisy environments. In: Proceedings of Sony Research Forum 1998. Tokyo (1998).

Kroch, A. Function and grammar in the history of english. In: (Fasold and Schiffrin, 1989), pp. 133-172.

Labov, W. Principles of Linguistic Change. Volume 1: Internal Factors. Oxford: Blackwell (1994).

Lyons, J. Language, Meaning and Context. Sussex, UK: Fontana (1981).

McIntyre, A. Babel: A testbed for research in origins of language (1998).

Meinhardt, H. Models of Biological Pattern Formation. London: Academic Press (1982).

Nichols, J. Linguistic Diversity in Space and Time. Chicago and London: The University of Chicago Press (1992).

Niyogi, P. and Berwick, R. The Logical Problem of Language Change. Tech. rep., MIT AI Laboratory and Center for Biological and Computational Learning, Department of Brain and Cognitive Sciences (1995). Technical Report AI Memo 1516/CBCL Paper 115.

Noble, J. Evolved signals: Expensive hype vs.ffconspirational whispers. In: Proceedings of Alife VI (Adami, e., ed.), pp. 358-367. Cambridge, MA.: The MIT Press (1998).

Oliphant, M. The dilemma of saussurean communication. Biosystems 1-2 (37), 31-38 (1996).

Pinker, S. The Language Instinct. London: Penguin Books (1994).

Prigogine, I. and Stengers, I. Order Out of Chaos. New York: Bantam Books (1984).

Ruhlen, M. On the Origin of Languages. Studies in Linguistic Taxonomy. Stanford, CA: Stanford University Press (1994).

Sebba, M. Contact Languages: Pidgins and Creoles. Basingstoke, UK: Macmillan Press (1997).

Steels, L. Self-organizing vocabularies. In: Proceeding of Alife $V$ (Langton, C., ed.). Nara, Japan (1996).

Steels, L. Constructing and sharing perceptual distinctions. In: Proceedings of the European Conference on Machine Learning (van Someren, M. and Widmer, G., eds.). Berlin: Springer-Verlag (1997).

Steels, L. and Kaplan, F. Stochasticity as a source of innovation in language games. In: Proceedings of Artificial Life VI (Adami, C., Belew, R., Kitano, H. and Taylor, C., eds.). Los Angeles: MIT Press (1998).

Steels, L. and Kaplan, F. Situated grounded word semantics. Tech. rep., Sony Computer Science Laboratory Paris (1999). Submitted to Publication.

Steels, L. and Vogt, P. Grounding adaptive language games in robotic agents. In: Proceedings of the 4 th European Conference on Artificial Life (Harvey, I. and Husbands, P., eds.). Cambridge, MA: The MIT Press (1997).

Thomason, S. and Kaufman, T. Language Contact, Creolization, and Genetic Linguistics. Berkeley, CA: University of California Press (1988). 